\title{
Appropriate Cluster Head Determination Strategy for extending the Wireless Sensor Network's (WSN's) Lifetime
}

\author{
V. Baby Shalini
}

\begin{abstract}
WSN is made with immense number of sensor's which are deployed densely over an unattended area is responsible for taking environmental measurements, process the data and finally transmit the sensed data over a wireless channel to the sink that makes decisions based on these sensor's readings. It is well known that the energy consumed for transferring one bit of data from individual sensor to sink is equivalent to a large number of arithmetic operations to be performed in a sensor processor. Thus, node clustering have been applied to hierarchical sensor networks with heterogeneity to augment the network existence whereas diminishing the necessary energy consumption. For that reason, Seven Level Balanced Energy Efficient Network Integrated Super Heterogeneous (SL-BEENISH) algorithm was designed and implemented. Simulation outcomes clearly demonstrated that SL-BEENISH attain better in comparison with TDEEC, BEENISH and IBEENISH with more stability period and network duration.
\end{abstract}

Keywords : Unattended, wireless, clustering, hierarchical.

\section{INTRODUCTION}

$W_{\text {ith the recent growth in micro electro mechanical }}$ systems (MEMS) technology field, cheap and multifunctional sensor's will exchange the information in diminutive distances have also happen to feasible. In general, sensor node [3] incorporates four essential units is exposed in Figure 1: a sensing unit, a processing unit, a transceiver unit and a power supply unit. The sensing unit consists of numerous sensor's intended to perceive the environment.

The processing unit incorporates a microprocessor which is in charge for power control over the sensor's, in addition to that it will process the perceived data and consequently make sure the communication with rest of the nodes present in the network. The transceiver unit comprises of short range radio employed to sustain incessant data transmission with left over sensor's. The power unit involves battery for supplying the power to the essential elements of the sensor. It is imperative to supervise the utilization of a battery power because if a mammoth current lasts for a lengthy occasion means, then the battery will be drained hastily. In such a

situation, cluster based techniques [1,2] play a significant role for accomplishing energy efficiency.

Revised Manuscript Received on December 16, 2019.

* Correspondence Author

V. Baby Shalini*, Department of Information Technology, Kalasalingam Academy of Research and Education, Krishnankoil, India. Email: v.babyshalini@klu.ac.in.
In clustering $[4,6]$, nodes come together into clusters with solitary node will be active as Cluster Head $(\mathrm{CH})$. The entire cluster members send out their sensed data to their pertinent $\mathrm{CH}$ which in turn forwards it to the far flung sink.

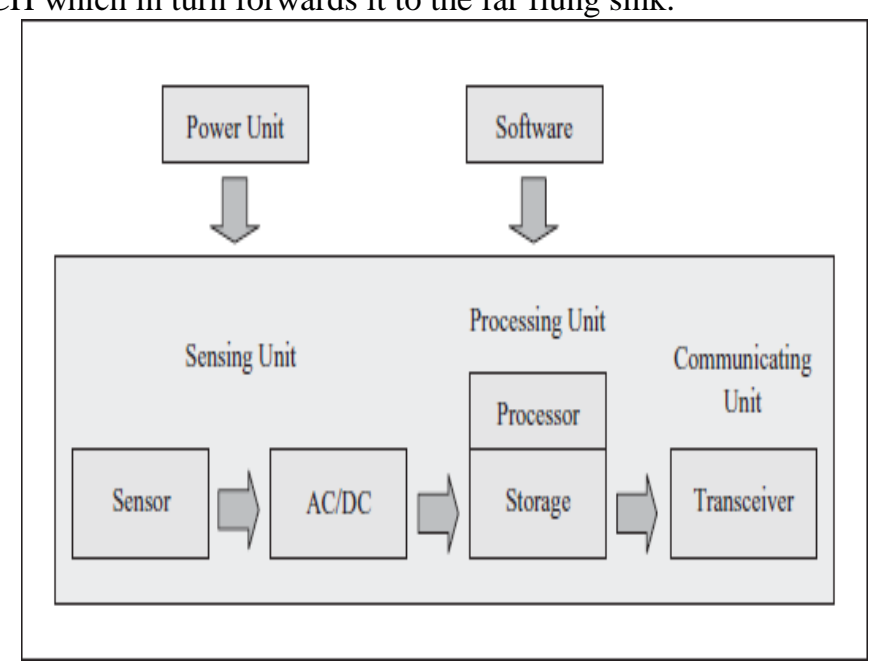

Fig. 1.Structure of Sensor's

This paper makes clear about seven level heterogeneous network model (SL-BEENISH) i.e normal, intermediate, advanced, super, ultrasuper, delux along with ultradelux. Here, CH's are chosen based upon proportion of nodes residual energy as well as network average energy. Ultradelux nodes are mostly favored to be designated as a CH's for the primary rounds, and when their energy declined to same level as that of normal ones, then delux nodes will have the prospect to become $\mathrm{CH}$ and so on. Therefore, energy is disseminated evenly over the sensor network. SL-BEENISH prolongs the network lifetime and stability period by heterogeneity [5].

\section{RELATED WORK}

Parul Saini et.al [7] introduced a cluster leader selection protocol named it as TDEEC in which network arrangement comprise of three different node types (normal, advance and super node), each node equipped with three different energy level. Here, $\mathrm{CH}$ is chosen based on the adjusted threshold value which is equal to proportion between a particular node remaining energy and network average energy of that round in respect to the optimum number of $\mathrm{CH}$.

N. Javaid et.al [8] obviously clarifies about BEENISH protocol in which four kinds of nodes (normal, advance, super and ultrasuper) are scattered in the sensing field. Here ultrasuper nodes have high energy when compared to super nodes and

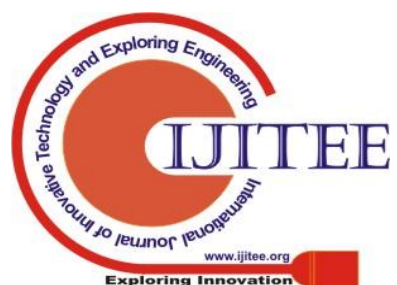


super nodes have high energy than advance nodes and advance nodes have high energy than normal nodes. The ultrasuper nodes are elected more as $\mathrm{CH}$ when compared to other nodes.

Harwinderpal Singh et.al [9] depicts about IBEENISH in which five types of nodes (normal, intermediate, advance, super and ultrasuper) are dispersed in the network. Here ultrasuper nodes have high energy than super nodes and super nodes have high energy than advance nodes and advance nodes have high energy than intermediate nodes and intermediate nodes have high energy than normal nodes. Among these nodes, ultrasuper nodes are elected more as $\mathrm{CH}$ when compared to other nodes.

\section{THE SL-BEENISH PROTOCOL}

Here, likelihood for $\mathrm{CH}$ selection is based on residual energy of sensor's $\left(E_{\text {resEnergy }}\right)$ as well as average energy of the network $\left(E_{\text {avgEnergy }}\right)$.

$E_{\text {avgEnergy }}$ of $\mathrm{r}^{\text {th }}$ round is calculated using the formula

$$
E_{\text {avgEnergy }}=\frac{1}{N} * E_{\text {TotalEnergy }} *\left(1-\frac{r}{N S}\right)
$$

$$
N S=\frac{E_{\text {TotalEnergy }}}{E_{\text {EnergyDissipated }}}
$$

Where $E_{\text {EnergyDissipated }}$ is the total energy spended by the network and is given by

$$
\begin{aligned}
& E_{\text {EnergyDissipated }}= \\
& L\left(N *\left(2 E_{\text {elec }}+E_{D A}\right)+k E_{m p} d_{t o S}^{4}+N E_{f s} d_{t o C H}^{2}\right)
\end{aligned}
$$

Where $k$ is the number of clusters, $d_{t o S}$ and $d_{t o C H}$ is the transmission distance from $\mathrm{CH}$ to $\operatorname{sink}(\mathrm{S})$ and $\mathrm{CM}$ to $\mathrm{CH}$ respectively and is calculated as

$$
\begin{aligned}
& k=\sqrt{\frac{N * E_{f s}}{2 \prod * E_{m p}}} * \frac{M}{d_{t o C H}} \\
& d_{t o S}=0.765 * \frac{M}{2} \quad d_{t o C H}=\frac{M}{\sqrt{2 \prod k}}
\end{aligned}
$$

The likelihood of normal, intermediate, advanced, super, ultrasuper, delux, ultradelux nodes in case of seven level heterogeneity are

Where $N S$ means network existence and is found using

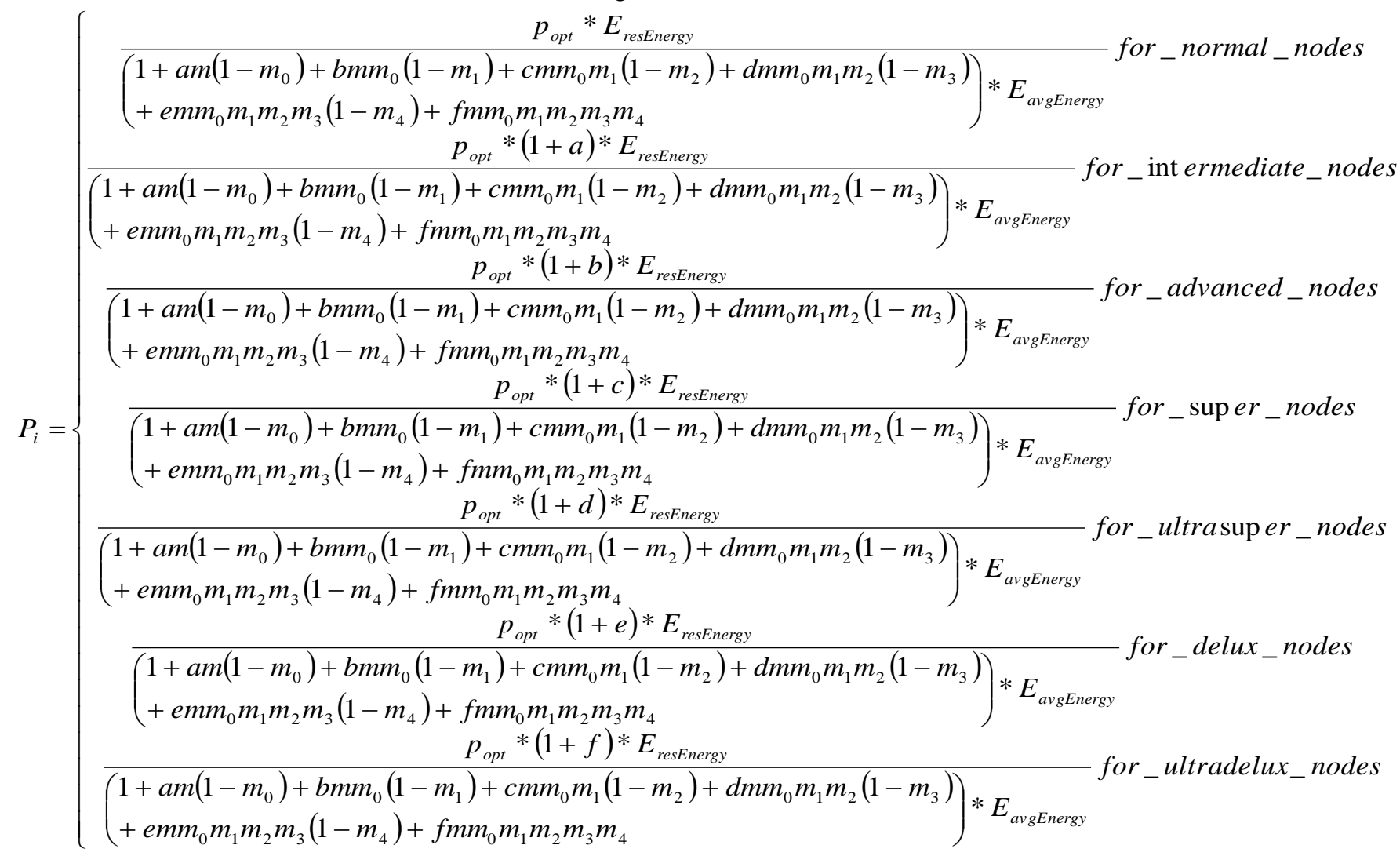


Each node use to determine whether itself to become a $\mathrm{CH}$ in each round, as follow

$$
T=\frac{P_{i}}{1-P_{i}\left(r \bmod \frac{1}{P_{i}}\right)}
$$

\section{RESULT AND DISCUSSION}

In this scenario, network model be composed of $\mathrm{N}$ sensor's hold seven dissimilar energy in $M \times M$ network field along with sink is placed centrally.

Here, 30 normal nodes which have $E_{\text {initial }}$ energy, 28 intermediate nodes include 1 time extra energy, 21 advanced nodes incorporate 1.5 times surplus energy, 13 super nodes have 2 times additional energy, 5 ultra super nodes contain 2.5 times superfluous energy, 2 delux nodes include 3 times additional energy, 1 ultradelux node have 3.5 times more energy when contrasted to normal nodes.

The parameters utilized for simulation purpose is delineated in Table I.

Table- I: Simulation Values

\begin{tabular}{|c|c|c|}
\hline Sl.No & Parameters & Values \\
\hline 1 & Overall Sensor's in the Region (N) & 100 \\
\hline 2 & $\mathrm{X}$ - Axis in the Monitoring Region (M) & 100 \\
\hline 3 & Y-Axis in the Monitoring Region (M) & 100 \\
\hline 4 & CH Prospect ( $\left.\mathrm{p}_{o p t}\right)$ & 0.1 \\
\hline 5 & Threshold Distance $\left(\mathrm{d}_{0}\right)$ & 80 meters \\
\hline 6 & $\begin{array}{l}\text { Energy Consumption happens at } \\
\text { Transceiver Electronics } \\
\left(\mathrm{E}_{\text {elec }}\right)\end{array}$ & $5 \mathrm{~nJ} / \mathrm{bit}$ \\
\hline 7 & $\begin{array}{l}\text { Energy Expenditure at the Free Space } \\
\text { Model } \\
\left(E_{f s}\right)\end{array}$ & $10 \mathrm{pJ} / \mathrm{bit} / \mathrm{m}^{2}$ \\
\hline 8 & $\begin{array}{l}\text { Energy Consumption at Multi Path Model } \\
\qquad\left(\mathrm{E}_{m p}\right)\end{array}$ & $\begin{array}{c}0.0013 \mathrm{~nJ} / \\
\mathrm{bit} / \mathrm{m}^{4}\end{array}$ \\
\hline 9 & $\begin{array}{l}\text { Energy Dissipation for Data } \\
\text { Cumulation }\left(\mathrm{E}_{D A}\right)\end{array}$ & $\begin{array}{l}\text { 5nJ/bit/ } \\
\text { Message }\end{array}$ \\
\hline 10 & Primary Energy $\left(\mathrm{E}_{O}\right)$ & $0.5 \mathrm{~J}$ \\
\hline 11 & Data Size to be Transmittal (L) & 4000 bits \\
\hline
\end{tabular}

Table- II: First Sensor Death

\begin{tabular}{|c|c|}
\hline Protocol & First Sensor Death (In Rounds) \\
\hline TDEEC & 1343 \\
\hline BEENISH & 1435 \\
\hline IBEENISH & 1570 \\
\hline SL-BEENISH & 1932 \\
\hline
\end{tabular}

First sensor death vs round number is revealed in Fig. 2. First node for TDEEC, BEENISH, IBEENISH and SL-BEENISH dies at $1343,1435,1570,1932$ respectively.

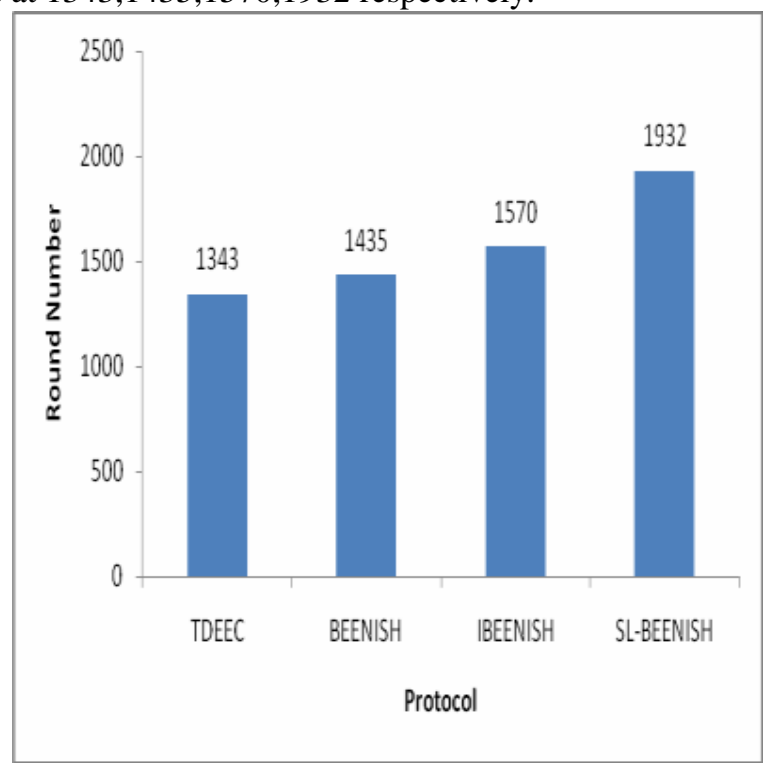

Fig. 2.First Node Death vs Round Number

Table- III: All Sensor Death

\begin{tabular}{|c|c|}
\hline Protocol & All Sensor Death (In Rounds) \\
\hline TDEEC & 7212 \\
\hline BEENISH & 8804 \\
\hline IBEENISH & 9654 \\
\hline SL-BEENISH & 9993 \\
\hline
\end{tabular}

All node dead vs round number is shown in Fig. 3. All node dead for TDEEC, BEENISH, IBEENISH and SL-BEENISH occur at 7212,8804,9654,9993 respectively.

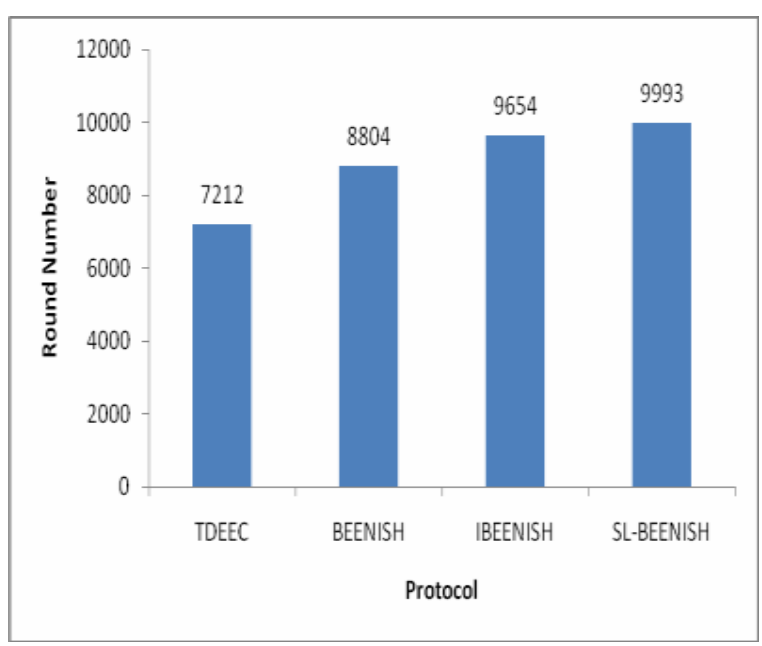

Fig. 3.Network Lifetime

\section{CONCLUSION}

Our proposed scheme SL- BEENISH uses the fraction of particular node left over energy and network average energy as CL selection criteria. Existing methods like TDEEC, BEENISH, IBEENISH and SL-BEENISH results are implemented and compared and then analyzed. From results, our proposed protocol extends the sensor network lifetime by an order of magnitude than other methods.

Published By:

Blue Eyes Intelligence Engineering \& Sciences Publication 


\section{REFERENCES}

1. V. Mhatre and C. Rosenberg, "Design Guidelines for Wireless Sensor Networks: Communication, Clustering and Aggregation", Ad Hoc Network, 2(1), 2004, 45-63.

2. A. A. Abbasi and M. Younis, "A Survey on Clustering Algorithms for Wireless Sensor Networks", Computer Communications, 30, 2007, $2826-2841$.

3. Carlos F. Garcia Hernandez, Pablo H. Ibarguengoytia Gonzalez, Joaquin Garcia Hernandez and Jesus A. Perez Diaz,'Wireless Sensor Networks and Applications: A Survey", International Journal of Computer Science and Network Security, 7(3), 2007.

4. D. Wei, Y. Jin, S. Vural, K. Moessner and R. Tafazolli, "An Energy Efficient Clustering Solution for Wireless Sensor Networks", IEEE Transactions on Wireless Communication, 10(11), 2011, 3973-3983.

5. F. A. Aderohunmu, J. D. Deng and M. K. Purvis, "Enhancing Clustering in Wireless Sensor Networks with Energy Heterogeneity", International Journal of Business Data Communication and Networking, 7(4), 2011, 18-32.

6. M. Ye, C. Li, G. Chen and J. Wu, "EECS: An Energy Efficient Clustering Scheme in Wireless Sensor Networks," International Journal of Ad Hoc \& Sensor Wireless Networks, 3, 2007, pp. 99-199.

7. Parul Saini and Ajay k Sharma, "Energy Efficient Scheme for clustering protocol prolonging the lifetime of heterogeneous wireless sensor networks", International journal of computer applications, 6(2), 2010.

8. T. N. Qureshi, N. Javaid, A. H. Khan, A. Iqbal, E. Akhtar, M. Ishfaq, "Balanced Energy Efficient Network Integrated Super Heterogenous Protocol for Wireless Sensor Networks", Procedia Computer Science, 19, 2013, pp 920-925.

9. Harwinderpal Singh and Lokesh Pawar," IBEENISH: Improved Balanced Energy Efficient Network Integrated Super Heterogeneous Protocol for Wireless Sensor Networks", International Journal of Computer Science and Network, 4(4), 2015.

\section{AUTHORS PROFILE}

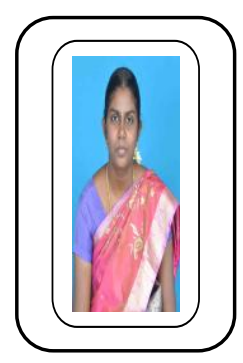

V. Baby Shalini is currently serving as assistant professor in department of Information Technology, Kalasalingam Academy of Research and Education, Krishnankoil..She received her Ph.D in 2019. She received her under graduate and post graduate degree in Electronics and Communication Engineering and Communication Systems from Anna University in 2006 and 2008 respectively. She has published few papers in Reputed International Journals and also a Lifetime member of Indian Society of Technical Education(ISTE). 